Int. J. Electrochem. Sci., 11 (2016) $7947-7959$

\title{
Azo Dye Decolorization in Bioelectrochemical System: Characteristic Analysis of Electrochemical Active Biofilms
}

\author{
You-Zhao Wang ${ }^{1, *}$, Ai-juan Zhou ${ }^{2}$, Yuan-Hua Xie ${ }^{l}$, Jin Han ${ }^{1}$, Mei-Yan You ${ }^{l}$, Min Wang ${ }^{1}$, Tong Zhu ${ }^{1, *}$ \\ ${ }^{1}$ Institute of Process Equipment and Environmental Engineering, School of Mechanical Engineering \\ and Automation, Northeastern University, Shenyang 110004, PR China \\ ${ }^{2}$ College of Environmental Science and Engineering, Taiyuan University of Technology, Taiyuan \\ 030024, PR China \\ *E-mail: wangyz@me.neu.edu.cn, tongzhu@mail.neu.edu.cn
}

doi: $10.20964 / 2016.09 .08$

Received: 15 May 2016 / Accepted: 30 June 2016 / Published: 7 August 2016

Electrochemical active biofilms (EABs) have attracted considerable attractions in bioelectrochemical systems (BESs) field for pollutants degradation from waste water. However, few studies have looked at EABs formation influenced by anode and cathode conditions. Therefore, EABs feature was investigated under different electrode conditions using a single-chamber BES. After EABs formation, the BES was effective in decolorizing the targeted pollutant azo dye, comparing to abio-electrode BESs. By analyzing the EABs microbial community compositions, anode EAB was dominated by a model electro-active Geobacter (29\%), whereas the cathode EAB microbial structure was relatively closer to the inoculum with low abundance Geobacter (2.5\%). This study suggested that changing the inoculum composition would be conducive to improving the cathode EAB capability for recalcitrant pollutant degradation in actual engineering applications.

Keywords: bioelectrochemical system; electrochemical active biofilm; azo dye; decolorizaiton

\section{FULL TEXT}

(C) 2016 The Authors. Published by ESG (www.electrochemsci.org). This article is an open access article distributed under the terms and conditions of the Creative Commons Attribution license (http://creativecommons.org/licenses/by/4.0/). 